\title{
Los estados del arte en ciencias sociales. Un camino para su escritura
}

\author{
The states of the art in social sciences. A way to write them \\ Os estados da arte em Ciências Sociais. Um caminho para sua \\ escritura
}

Javier Duque*

\begin{abstract}
RESUMEN
El presente artículo sobre los estados del arte plantea una reflexión sobre su sentido, describe sus dimensiones y sugiere una estrategia para su escritura. Es un artículo de metodología en ciencias sociales. El argumento central es que, a través de los estados del arte se prepara el camino de la investigación y se evitan los seudoproblemas, las reiteraciones y la circularidad, frecuentes en las ciencias sociales, especialmente en América Latina. Aunque mucha investigación produce conocimiento nuevo, también son frecuentes las reiteraciones y esto se evita si se tiene claridad acerca del estado del conocimiento respecto de la temática objeto de la investigación. De acuerdo con esto, se hace una propuesta sobre cómo escribir los estados del arte de una forma sistemática y fructífera.
\end{abstract}

\section{SUMMARY}

This article on the states of art proposes a reflection on its meaning, describes its dimensions, and suggests a strategy for its writing. It is an article on methodology in social sciences. Through the states of the art, the central argument is that the path of research is prepared, and we avoid pseudo-problems, reiterations, and circularity, frequent in the social sciences, especially in Latin America. Although much research produces new knowledge, reiterations are also frequent. This can be avoided if there is clarity about states of the art regarding the research's subject matter. Ac-
Palabras clave: educación, estados del arte, investigación, metodología, teoría.

Key words: education, states of the art, research, methodology, theory. 
cordingly, a proposal is made on how to write the states of the art systematically and fruitfully.

\section{RESUMO}

O presente artigo sobre os estados da arte propõe uma reflexão

Palavras-chave: educação, estados da arte, pesquisa, metodologia, teoria. prepara-se o caminho da pesquisa e são evitados os pseudoproblemas, as reiterações e a circularidade, frequentes nas Ciências Sociais, especialmente na América Latina. Apesar de muita pesquisa produzir conhecimento novo, também são frequentes as reiterações e isto é evitado quando se tem claridade sobre o estado do conhecimento com relação ao tema objeto da pesquisa. De acordo com isto, faz-se uma proposta sobre como escrever os estados da arte de uma forma sistemática e frutífera. 


\section{Introducción}

En otros tiempos y en numerosos manuales de metodología de investigación se solía considerar, en alguna parte de los esquemas o guías que (se supone) permitían a los estudiantes adelantar con éxito un trabajo de grado o una tesis, un apartado que se denominaba "antecedentes", esta expresión fue cambiada luego por "balance bibliográfico", "revisión de la literatura" o, con más pretensiones, por "revisión crítica de la literatura". Estos términos aludían a una búsqueda, inventarios y comentarios acerca del tema que se estaba indagando y respecto del cual se estaba incursionando y recopilando información. El propósito inicial era incursionar en una temática, hacer un primer ejercicio de búsqueda, presentar un panorama general de lo que se había escrito sobre el tema o sobre otros temas conexos. Aunque era preliminar, solía ser incluido en los rígidos esquemas de presentación de trabajos de grado y tesis, como un apartado después de formular y plantear el problema de investigación.

Posteriormente se fueron generalizando las expresiones "estados del arte" y "estados de la cuestión". La primera es la traducción de la expresión en inglés "state of the art", que hace referencia al nivel más avanzado de desarrollo alcanzado en un momento determinado en cualquier ciencia, técnica o campo específico de conocimiento (Macmillan Dictionary, s.f). La segunda expresión ha corrido paralela a la primera y es más usada en español.

Los estados de la cuestión son ejercicios académicos sistemáticos orientados a "usar el pasado y el presente para pensar el futuro" (Torraco, 2016). Recurren a la literatura, a la bibliografía existente sobre un tema para conectarla entre sí, ordenarla en virtud de determinados criterios y reconstruir un panorama de artículos, libros, capítulos de libros, ponencias, memorias, que se ponen en escena conectadas y comunicadas entre sí para derivar hacia nuevas búsquedas. Es una labor de revisión-evaluación de lo que existe para proyectarse hacia lo nuevo, resultado del diagnóstico de aquello que falta, que no está bien propuesto, que denota falencias, que es incompleto, que no persuade, que inquieta.

Estos documentos son el resultado de dos actividades académicas diferentes. Por una parte, de la labor de un académico, un investigador 
experimentado que hace y escribe una revisión profesional de literatura y deriva de esta una serie de problemas, ya sea que esté conectada con un proyecto específico o que proponga a sus colegas un panorama sugerente de nuevas cuestiones. Examinan el alcance, el rango y la naturaleza de las actividades de investigación en un área en particular, revisiones muy completas de la bibliografía que permiten identificar brechas de investigación. De acuerdo con su objetivo principal, estas revisiones concluyen con la presentación de una agenda de investigación detallada para trabajos futuros (Paré \& Kitsiou, 2017). Por otra parte, también son ejercicios académicos realizados por estudiantes que están en el proceso de elaboración de un trabajo de grado, y que adelantan una exploración sistemática sobre una temática de interés, de la cual esperan confluir en un proyecto a partir de la formulación de un problema.

El presente artículo propone una forma de construir los estados de la cuestión y parte de considerar que estos constituyen una vía para evitar la reiteración sistemática, como uno de los obstáculos para el avance de las ciencias sociales en nuestros contextos. Este tipo de saberes tiende a ser muy reiterativos y circulares, y tanto la investigación profesional como la formativa, que se adelanta con los estudiantes universitarios con mucha frecuencia, redundan, reiteran, repiten, vuelven a decir lo dicho, sintetizan, resumen. Aunque las revisiones críticas sobre temas y problemas son ejercicios académicos válidos, la investigación debe evitar reproducir la práctica de la circularidad y la repetición, y explorar nuevas posibilidades, nuevas realidades, aplicar teorías a espacios inéditos, proponer nuevos ámbitos, nuevas aristas, nuevos puntos de vista. Esto es más factible si se conoce en profundidad el panorama del conocimiento existente y los estados de la cuestión son fundamentales para lograrlo.

No sobra advertir que se trata de un artículo sobre metodología. Esto no implica una concepción procedimental tipo "metodolatría", que considera que el proceso investigativo corresponde a caminos inexorables, rutas preestablecidas que, seguidas como recetarios, permiten realizar investigaciones. Es claro que la investigación social es compleja, que hay diversos caminos, estrategias variadas, procedimientos múltiples, y que no es posible encapsularla como suelen hacer algunos manuales de metodología. 
El artículo consta de tres partes. La primera define a los estados de la cuestión y enfatiza en la idea de que estos constituyen un punto de partida, por cuanto sin un conocimiento adecuado de la temática no es posible problematizarla. La segunda parte trata de las cuatro coordenadas que corresponden a las dimensiones teórica, conceptual, metodológica, y a los resultados de los estudios que son revisados y analizados. La tercera parte propone una forma de hacer los estados de la cuestión, con sugerencias de instrumentos de búsqueda y sistematización de la bibliografía.

\section{Los estados de la cuestión como punto de partida}

Los estados de la cuestión han sido definidos de diversas formas. De manera escueta, se consideran como una "revisión de antecedentes" o como "investigación de investigaciones" (Abreo, 2004), o como una labor de "inventariar y sistematizar la producción en determinada área de conocimiento" (Garcés, Patiño y Torres, 2008). También se considera como "una investigación documental sobre la cual se recupera y trasciende reflexivamente el conocimiento acumulado sobre determinado objeto de estudio (...) que pretende hacer explícita la postura teórica y metodológica de los diferentes estudios" (Galeano y Vélez, 2002). Aluden estas definiciones al hacer acopio, a recopilar, a darle una mirada a lo que se ha hecho y presentarlo e inventariarlo, por lo regular de forma cronológica.

Otras formas de entender los estados de la cuestión consideran que no solo se trata de recopilar, revisar y ordenar la producción académica sobre un tema, también son asumidos como un punto de partida para saber qué camino seguir en un proceso de indagación. Se plantea que tienen el propósito de encontrar y mostrar vacíos, tendencias y nuevas perspectivas teóricas para adelantar otras investigaciones (Arboleda y Zabala, 2005). Se considera que estos proveen una síntesis crítica del estado de conocimiento, pero también permite establecer brechas en el conocimiento e identificar nuevos problemas (Schrye, Wagner \& Benlian, 2015). En tal sentido, se define como "una investigación sobre la producción investigativa de un determinado fenómeno. Este permite develar la dinámica a partir de la cual se ha desarrollado la descripción, explicación o comprensión del fenómeno en estudio y la construcción de conocimientos sobre el saber acumulado" (Uribe, 2005). 
Una forma sintética e integral de asumir los estados de la cuestión es considerar que estos deben cumplir con tres criterios: sintetizar los resultados de investigaciones anteriores sobre el mismo tema; identificar vacíos, limitaciones y aportes en los trabajos previos, y proporcionar un marco para nuevas indagaciones (Boote \& Beile, 2005). Los estados de la cuestión tienen dos propósitos: presentar hasta dónde se ha llegado en las investigaciones, reflexiones y análisis sobre un tema específico, y hacerlo con la perspectiva de poder avanzar, de diagnosticar nuevas perspectivas, aquello que falta, los vacíos, lo que no se ha dicho. Una definición que incluye ambos aspectos de forma clara es la siguiente:

El estado del arte organiza y compendia la construcción teórica de una investigación y se centra en el problema, los objetivos, la metodología y los supuestos resultados, dejando de manifiesto la novedad del campo de conocimiento específico (nuevos conocimientos, evitando la repetición y asegurando la originalidad en el aporte técnico y la significación práctica). El estado del arte/técnica, deja en relieve el objeto de estudio, evaluando las principales tendencias encontradas durante la revisión bibliográfica. (Urbina y Morel, 2017, p. 2)

Así, con los estados de la cuestión se apunta a clarificar qué se ha dicho (contenido, argumentos, tesis), en qué disciplinas fue dicho (es frecuente que un tema sea abordado por diversas disciplinas); cuándo fue dicho (año, época, momento, periodo), en dónde fue dicho (en libros, artículos, conferencias, ponencias, tesis), cómo fue dicho (la metodología, las fuentes utilizadas). Por supuesto, como no se trata solo de una revisión-balance, es importante también determinar lo que no se ha dicho, lo que falta por abordar, lo que se ha dicho y no ha generado acuerdos o es muy debatido, aquello que se ha dicho, pero deja zonas sin explorar (Calvo y Castro, 1995; Jiménez, 2006).

De este doble contenido de los estados de la cuestión pueden resultar dos productos diferentes. Pueden ser una investigación en sí misma, un ejercicio académico sobre un tema con el propósito de presentar un panorama sobre los enfoques, las metodologías, las fuentes, los resultados, así como los vacíos, las deficiencias, los debates, y las cuestiones pendientes. En algunos casos se suelen denominar "revisiones siste- 
máticas" de un tema, cuyos alcances dependen de los objetivos concretos que se hayan planteado. En el caso de la investigación profesional, suele orientarse a demostrar que el conocimiento alcanzado hasta el momento no es el adecuado, deja vacíos, tiene zonas poco cubiertas o requiere de nuevos planteamientos. También pueden hacer parte de un proyecto de investigación, un "momento" en su formulación, de cuyo contenido se desprenden retos, preguntas y cuestiones que van a ser indagadas. En tal sentido, constituye una especie de "diagnóstico" que sirve para detectar aquello sobre lo cual se ha dicho poco, no se ha dicho nada, se ha dicho de forma insuficiente, se ha dicho de forma cuestionable o no se comparte alguna parte de lo que se ha dicho.

Es importante enfatizar que, mientras en las ciencias naturales se da por entendido que los estados de la cuestión permiten básicamente establecer un balance para determinar el conocimiento establecido y no "descubrir lo descubierto", en ciencias sociales es muy frecuente la redundancia y la reiteración; por ello, se debe tener claro que, además del ejercicio de reconstruir un panorama de lo que se ha indagado y escrito sobre un tema, el objetivo central de esta "investigación dentro de la investigación" es establecer aquello que no se ha hecho, lo que no se ha dicho, para evitar reiteraciones, duplicar esfuerzos para llegar al mismo lugar o partir de lo conocido para repetirlo, decirlo de otra forma, incluso repitiendo errores, presunciones, especulaciones y afirmaciones sin una red amplia de evidencias empíricas.

Como lo expresan Gay King, Robert O. Kehone y Sidney Verba (2000), es necesario situar el diseño del estudio dentro del marco de la bibliografía existente, lo cual garantiza que el investigador comprende lo que se ha hecho y los avances en la temática que está abordando, y se reduce así la posibilidad de repetir lo que se ha dicho y, en cambio, que se puede hacer una aportación al tema. Los autores sugieren algunos caminos para hacer aportes a lo ya existente:

(a) Elegir una hipótesis que se considere importante dentro de la bibliografía existente de la cual no se ha realizado un estudio sistemático. Se trata de encontrar pruebas a favor o en contra de esta hipótesis. 
(b) Elegir una hipótesis aceptada en la bibliografía, pero de la cual hay recelos y algunos cuestionamientos. Se trata de ver su validez en algún estudio de caso.

(c) Mediante un análisis de caso o comparado, presentar más pruebas a favor de una teoría que está en disputa con otras teorías. Es decir, adoptar una teoría que persuada con sus argumentos y amplíe su validez con la indagación.

(d) Adelantar una indagación que pueda cuestionar hipótesis que generalmente son aceptadas, pero que en estudios específicos no parecen tan válidas.

(e) Plantear que en la bibliografía existente no se ha prestado atención a un asunto que se considera importante y se propone hacer algún aporte.

(f) Aplicar teorías de un subcampo de conocimiento a otro, adaptando así sus aportes, aunque en apariencia parecen lejanos a la disciplina.

Aunque suele plantearse que los estados de la cuestión suponen una previa formulación del problema de investigación y de los objetivos, consideramos que es al revés. Si el propósito del estado de la cuestión es hacer un balance crítico y lo más exhaustivo posible acerca de lo que se ha adelantado sobre el tema, las interrogantes que van a orientar la investigación son el resultado de este ejercicio solo cuando se ha realizado un diagnóstico, y se sabe qué, cómo, cuándo y dónde se han hecho investigaciones sobre este. En gran parte, las tendencias reiterativas de los trabajos en ciencias sociales se derivan de un inadecuado conocimiento de la bibliografía sobre el tema, aunque también se relacionan con los niveles de institucionalización de la respectiva ciencia social en el país, por cuanto un bajo nivel de desarrollo de la disciplina se expresa en las frecuentes compilaciones y artículos - incluso libros- que solo resumen o sintetizan la bibliografía existente. Los llamados "trabajos bibliográficos", tan extendidos en muchas universidades, son ejercicios de este tipo. Estos son válidos y cumplen una función formadora en ciertos niveles, pero si se convierten en algo habitual, frecuente y no trascendidos hacia la producción de nuevo conocimiento, se constituyen en una traba para el avance de la disciplina. Incluso se llega a 
confundir esta labor de reiteración organizada de la bibliografía con la investigación.

La demarcación progresiva de una temática objeto de indagación la representa Holger R. Maier (2013) mediante la figura de una pirámide invertida. Parte ésta de la definición del dominio temático del cual se va a ocupar la indagación. Todas las ciencias sociales cuentan con subcampos, o áreas o especialidades, y un paso inicial básico consiste en identificar, de forma clara, en cuál de estos se ubica la temática de interés. Aunque sigue siendo un ámbito muy comprensivo y amplio, constituye un paso inicial de recorte, de demarcación de lo que será el objeto de estudio, y esto se reflejará en la cobertura que tendrá la búsqueda de la bibliografía. Luego sigue una adecuada identificación de estudios relevantes que permitan tener un panorama adecuado de lo que se ha hecho y dicho sobre el tema, y se va abordando la discusión crítica de la literatura dentro de este dominio. Esto permite detectar vacíos, plantear nuevos problemas, identificar necesidades de investigación y las brechas de conocimiento dentro de la literatura. Tras su realización se está en condición de perfilar de forma clara los objetivos de investigación, que deben abordar una o más de las brechas de conocimiento y las necesidades de investigación identificadas como el resultado de la revisión crítica de la literatura.

Esta pirámide invertida describe bien la lógica general de la construcción de los estados de la cuestión y advierte sobre lo que no es este ejercicio académico: no es un inventario de bibliografía general, dado que tiene como referente una o varias disciplinas y los subcampos específicos; no es la presentación ordenada de lo que dicen algunos estudios: implica el análisis de su contenido, los aportes que se han hecho, así como los vacíos y las brechas de conocimiento; no es un requisito formal posterior a la formulación de un problema, sino un requisito esencial para formularlo de forma adecuada; no es un escrito para reafirmar que el trabajo en ciernes ya lo han hecho otros y eso lo legitima: por el contrario, al resaltar los estudios previos, se constata que hay otros propósitos, nuevos propósitos. 


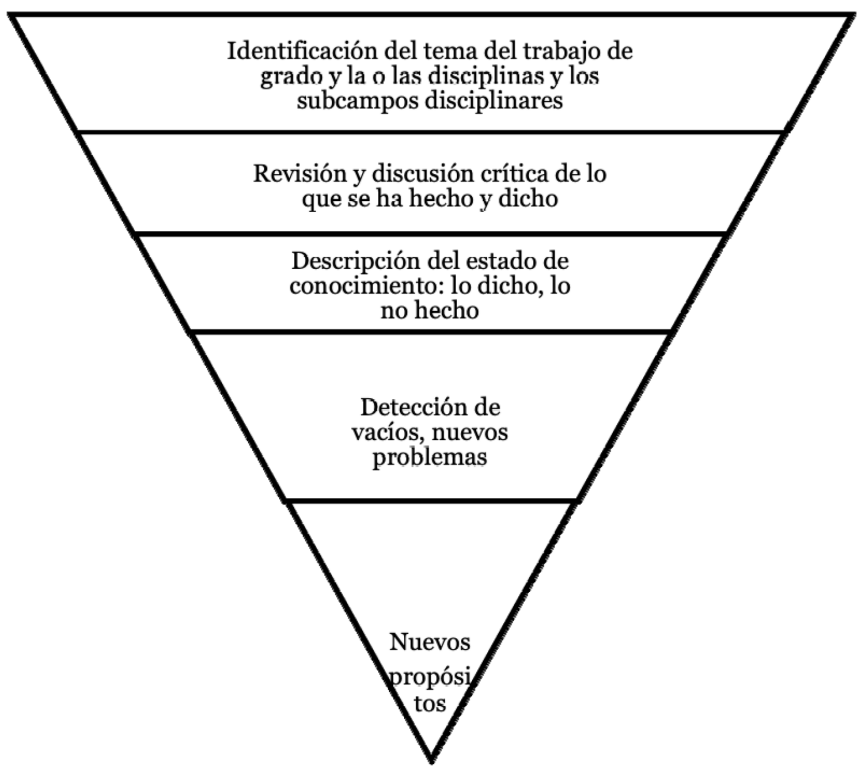

Gráfico 1. La pirámide invertida de los estados de la cuestión deHolger Maier (2013)

En suma, lo que se afirma sobre las revisiones críticas de la literatura es válido también para los estados de la cuestión y, después de haberlos realizado, se debe tener una idea clara de: (i) los principales logros en el campo revisado (qué se ha hecho, qué aportes hay, cuál es el estado de desarrollo de las indagaciones y reflexiones sobre el tema); (ii) las principales áreas de debate (en ciencias sociales, en particular en ciencia política, siempre hay visiones diferentes y a veces confrontadas sobre los temas), y (iii) cuáles son las preguntas de investigación que podemos detectar, derivar o formular (no es solo inventariar, comentar y analizar lo existente, también visualizar las perspectivas nuevas, caminos de exploración, nuevas búsquedas) (Pautasso, 2013).

\section{Los componentes de los estados de la cuestión}

Los estados de la cuestión son más que un registro ordenado de la bibliografía precedente, son más que inventarios y descripciones de trabajos anteriores, y requieren de una elaboración más compleja. Suponen buscar y sistematizar información, hacer lecturas complejas y críticas, ordenar y construir conceptos, diferenciar teorías, conocer y 
saber utilizar metodologías. Además, la construcción de un estado de la cuestión es una oportunidad más de aprendizaje y de formación, orientada al manejo de las coordenadas de referencias básicas sobre la temática que se indaga para un trabajo de grado. Los estados de la cuestión permiten dar cuenta de ¿cuáles son los estudios más relevantes y representativos sobre la temática de nuestro interés? ¿Cuáles teorías o enfoques analíticos han sido utilizados en las investigaciones? ¿Cuáles son los alcances y limitaciones de estas teorías? ¿Qué conceptos se han retomado y/o construido en los estudios analizados? ¿Qué estrategias metodológicas han sido utilizadas en estos estudios y cuáles son sus potencialidades y limitaciones? ¿Qué tipo de información y a qué fuentes han recurrido los estudios? ¿Cuáles son los resultados que despiertan más consensos y cuáles los que producen más debates y cuestionamientos?

Los estados de la cuestión permiten tener un panorama general de los estudios previos sobre la temática y familiarizarse con conceptos, teorías, metodologías y contenidos. La mayor cercanía con el tema de estudio facilita al investigador identificar las áreas generales de interés, los temas específicos que han sido estudiados, las áreas de énfasis, las más desarrolladas y las menos exploradas. La revisión también ayuda a comprender las interrelaciones entre el tema que se está considerando y otras áreas temáticas, y abre espacio a nuevos problemas de indagación. Como se trata de un ejercicio de reconstrucción, es frecuente -y en muchos casos conveniente- incluir una perspectiva histórica, la comprensión de los orígenes del interés académico, cómo se ha desarrollado la investigación sobre la temática a lo largo del tiempo, el origen de los enfoques y teorías, la evolución de los énfasis y metodologías a partir de las cuales ha sido abordada la temática (Hart, 1999).

En su dimensión teórica, este ejercicio permite describir y evaluar las teorías utilizadas en los estudios sobre la temática; asimismo, las críticas que se han hecho a estas, sus alcances, sus méritos y sus limitaciones. En general, en las ciencias sociales actuales hablamos de múltiples teorías y estas son de alcance medio, por cuanto no hay alguna que pretenda tener un alcance general, tipo "gran paradigma". Por ello, en el proceso de lectura, análisis, síntesis y registro que precede a la escritura de un estado de la cuestión, una labor central corresponde a la identificación de los enfoques teóricos en que se fundamentan los 
estudios que se están revisando, sus argumentos y premisas. Una posición crítica conlleva conceder que un enfoque o punto de vista existente tiene algunos méritos, y que puede ser útil en el análisis, pero que otros necesitan ser revisados, que tienen debilidades o, incluso, que no son aceptables por su falta de fundamento, de claridad, de coherencia.

Puede resultar de esta búsqueda y análisis de las teorías disponibles la detección de controversias, algunas del pasado y otras vigentes. Dar cuenta de un debate tiene relevancia en sí mismo, no solo en términos de historia de las ideas, sino también por las derivaciones que pudo haber tenido, al cuestionarse un enfoque, una manera de entender el mundo y la realidad social. Asimismo, del examen a las teorías se desprende una reflexión acerca de su validez y su capacidad explicativa, así como su utilidad para abordar nuevos problemas (Baumeister \& Leary, 1997). Esto, en sí mismo, resulta ser un hallazgo y, a la vez, un reto que es necesario asumir, y las alternativas pueden ir desde limitar el trabajo de grado a un nivel descriptivo, evitando entrar en la controversia (lo cual es válido), convertir esta situación en el problema de indagación (producir un texto de debate sobre la situación actual de las teorías en la investigación sobre la temática, sus alcances y limitaciones), o intentar la vía de la combinación no contradictoria de elementos de dos o más teorías, manteniendo la apertura al diálogo y rehusando el camino de los dogmatismos y, más aún, de las militancias ideológicas revestidas de academia.

En su dimensión metodológica, los estados de la cuestión se orientan a identificar y analizar las metodologías y técnicas utilizadas en la investigación, y las reflexiones acerca de la temática, además de examinar sus ventajas y desventajas: cómo constriñen o posibilitan los alcances de los estudios, cuáles son sus deficiencias y limitaciones. Hay que analizar si los textos revisados y analizados recurrieron a metodologías cualitativas, cuantitativas o mixtas, si fue utilizado material histórico primario, si se trata de estudios de caso o estudios comparados, etnografía, análisis documental, estudios basados en estadística inferencial, qué tipos de fuentes sirvieron para sustentar los argumentos, si fueron suficientes y convincentes los resultados, si fueron apropiadas las técnicas utilizadas para identificar, recopilar y analizar los datos. En particular, es conveniente estar atento para ver si los textos considerados cuentan con una suficiente red de evidencias empíricas y están 
claramente reseñadas las fuentes de estas. No siempre las metodologías se plantean de forma explícita y es necesario descifrarlas, incluso en algunos textos estas pueden ser confusas o poco convincentes, poco claras y rigurosas, y esto hay que advertirlo para no repetir los mismos errores.

En cuanto a los argumentos y resultados del análisis, es necesario considerar su solidez, coherencia y claridad, pero también mostrar los vacíos en el razonamiento, el uso de la evidencia, la fuente de las evidencias, la influencia de las suposiciones o la lógica de las interpretaciones que se han hecho, y las consecuencias lógicas de las conclusiones. Se ha sugerido considerar si los textos analizados contienen una estructura explícita, clara y confiable; si se definen de forma cuidadosa los conceptos utilizados; si se presentan las razones válidas para incluir información, fuentes, documentos (si todas las evidencias son documentadas y confiables); si los argumentos no están atravesados por juicios de valor (considerar si los autores mantienen y respetan normas de imparcialidad en el análisis); si se plantean generalizaciones sustentadas o si, por el contrario, se recurre a falacias argumentativas; si se recurre o no a criterios de autoridad, de tradición o a lugares comunes dados como válidos, sin fundamentos (Hart, 1999).

Finalmente, es importante considerar la legitimidad de los contenidos de la bibliografía revisada, así como el resultado del propio estado de la cuestión. Para evitar críticas a su revisión, debe utilizar sus fuentes correctamente. Se espera que las fuentes revisadas -y el escritono violen los estándares y valores del trabajo académico. Para ello hay una serie de acciones a considerar: no se tergiversan los argumentos de los demás autores; no se registran las especulaciones como si fueran hechos; se cita de forma adecuada, guardando la fidelidad a las fuentes, y se cita de forma tal que sea fácilmente rastreable la fuente; además, se revela y denuncia cuando se detectan plagios (Hart, 1999).

En suma, la revisión-balance-evaluación debe permitir establecer el conocimiento acumulado, los acuerdos y convergencias en la bibliografía sobre el tema y, de igual forma, los desacuerdos y divergencias. No solo se señalan los aportes de la bibliografía, también es necesario subrayar los vacíos, las zonas grises, lo que no se ha dicho o se ha dicho poco, o de forma poco convincente. Se evalúa críticamente el material que ya se ha publicado, a la vez que se abre el camino para ubicar el tra- 
bajo y se plantea cuál va a ser su alcance. El resultado de un estado de la cuestión integral, que incluya las dimensiones teórica, conceptual, metodológica y del desarrollo de aportes-vacíos, debe permitir responder ¿cuál es la estructura del conocimiento sobre este tema? ¿Cuáles y quiénes son las obras clave y los teóricos? ¿Qué supuestos metodológicos han servido de base a los trabajos previos y se consideran necesarios para estudiar este tema? ¿Cuáles de las tesis del autor son más convincentes o menos convincentes? ¿Contribuye de alguna manera significativa a una comprensión del tema?

\section{Una manera de hacer los estados de la cuestión}

Como se planteó, los estados de la cuestión buscan, por una parte, dar cuenta, de una forma sistemática y lo más completa posible, de lo que se ha dicho sobre el tema de indagación (contenido, argumentos, tesis, teorías), en qué disciplinas fue dicho (es frecuente que un tema sea abordado por diversas disciplinas); cuándo fue dicho (año, época, momento, periodo), dónde fue dicho (en libros, artículos, conferencias, ponencias, tesis), cómo fue dicho (la metodología, las fuentes utilizadas). Por otra parte, al elaborar una especie de "diagnóstico" sobre el tema, sirve para detectar aquello sobre lo cual se ha dicho poco; sobre lo que no se ha dicho nada -que suele ser una excepción-; aquello que se ha dicho de forma insuficiente y ha dejado muchos vacíos; lo que se ha dicho de forma cuestionable, sin evidencias suficientes; lo que se ha dicho y reiterado, pero sobre lo cual no se comparte lo dicho.

¿Cómo hacerlos? ¿Cómo adelantar de buena forma esta indagación bibliográfica, de tal forma que permita adelantar esta doble labor de revisión analítica y detección de problemas? Y, sobre todo, recuperando un sugestivo título de un artículo “ ¿Cómo navegar hoy por el tsunami de la información: sobre el arte de la lectura efectiva”? (Erren, Cullen \& Erren, 2009).

Un camino para esta labor puede partir de considerar lo que busca el estado de la cuestión. Pippa Hemingway plantea que un ejercicio de revisión de alta calidad respecto de la literatura sobre un tema - que podemos hacer extensiva a los estados de la cuestión- busca básicamente seis cosas: (a) identificar estudios relevantes publicados y no publicados (ya esto representa un criterio de demarcación importante, por cuanto puede haber centenares o miles de artículos y libros, y es 
fundamental ser selectivos, no con criterios subjetivos, de preferencias teóricas o de simpatías hacia algún autor, sino con base en criterios objetivos, como los que establecen los estudios bibliométricos, que contabilizan las citas, identifican a los autores y las obras más citadas y/o de mayor influencia, o, mediante la identificación de los que son considerados autores y obras clásicas, obras pioneras, obras que son referentes obligados); (b) seleccionar estudios o informes para su inclusión y hacerlo de forma meticulosa (puede hacerse la selección teniendo en cuenta las fuentes, por ejemplo, solo lo publicado en revistas incluidas en indexaciones reconocidas nacional e internacionalmente); (c) evaluar la calidad de cada estudio o informe (a la vez que establecer un criterio de exclusión: aquellos textos que, por su extensión, modalidad, desarrollo, calidad, no van a ser incluidos); (d) sintetizar los resultados de estudios o informes individuales de una manera imparcial; (e) interpretar los hallazgos; (f) presentar un balance y resumen de estos.

Teniendo en mente lo anterior, sugerimos un posible camino a seguir en la escritura de un estado de la cuestión.

I. Tener claridad sobre la temática y sobre su demarcación. Es fundamental tener claridad sobre la temática, focalizada y definida, como se planteó en la descripción del triángulo invertido. La búsqueda inicial es conceptual-céntrica, es decir, está ligada directamente a los conceptos relacionados con la temática del trabajo de grado. Como ya se planteó, no se trata de la o las preguntas de investigación, que son el resultado del balance que se realice y de la detección de los vacíos, las cuestiones no abordadas, las abordadas que son muy parciales o incompletas, los casos en los que el saber existente no ha puesto atención o no ha tenido la cobertura. Como actualmente hay centenares, miles de fuentes sobre cualquier tema, es necesario hacer recortes y demarcaciones de la temática que sean equilibrados: no ser tan general que haga que en la búsqueda quepa todo, ni tan específico que impida encontrar bibliografía.

II. Determinar cómo se van a detectar e identificar las fuentes de información. Hay muchas fuentes de información a considerar y, en la actualidad, cualquier búsqueda sobre casi cualquier tema puede arrojar miles de referencias y se corre el riesgo de perderse en el mar si no se cuenta con la orientación básica inicial que permita navegar sin naufragar y quedar al garete. ¿Por dónde comenzar? En un comienzo 
se puede considerar tres recursos: las bibliografías especializadas contenidas en las bases de datos de internet; la búsqueda manual de fuentes en bibliotecas, resúmenes, bibliografías de libros y artículos clave, y el concepto de expertos.

La búsqueda de bases de datos electrónicas es a menudo el punto de partida principal, pero a estas es necesario entrar demarcando, recortando y seleccionando, teniendo en claro la cobertura en el tiempo, el tema y subtemas, y las fuentes que se van a consultar y las que no. Hay que focalizar la búsqueda en la temática, su espacialidad y temporalidad, y recurrir a las palabras clave de las revistas, a descriptores y usar diferentes fuentes de base de datos. Asimismo, buscar tesis y trabajos de grado en las universidades (esto se facilita cada vez más, porque las universidades están adoptando la política de conocimiento abierto y se publican en su versión original online). Hay una herramienta especialmente valiosa y la proporciona las bibliografías de Oxford Online (www.oxfordbibliographies.com/). En estas se presenta una recopilación de la bibliografía que consideran más relevante sobre un tema y que son elaboradas por expertos reconocidos por la comunidad académica. Cada autor da cuenta del tema identificando a los investigadores más importantes y sus publicaciones relacionadas con el tema, también se incluyen las referencias bibliográficas con breves comentarios.

Algunas bases bibliográficas en ciencias sociales disponibles en internet son: Bibliografía internacional de las ciencias sociales: una enorme base de datos de bibliografía de ciencias sociales de revistas, libros y capítulos de libros, en varios idiomas y publicados desde 1951 hasta la actualidad. Sociofile database: contiene resúmenes sociológicos de aproximadamente 2.000 revistas (publicados desde 1974 hasta la actualidad). También es importante Sociological Abstract, una base de datos de resúmenes de sociología y ciencias del comportamiento, de Cambridge Scientific Abstracts. Incluye libros, capítulos de libros, disertaciones y ponencias de congresos. Los resúmenes de servicios sociales brindan cobertura bibliográfica de la investigación actual centrada en el trabajo social, servicios humanos y áreas relacionadas, incluido el bienestar social, la política social y el desarrollo comunitario. La base de datos incluye resúmenes de artículos de revistas y disertaciones, así como citas de reseñas de libros (www.proquest.com/pro- 
ducts-services/socioabs-set-c.html). Social Sciences Citation Index es una base de datos de resúmenes y referencias citadas de más de 1.700 revistas de ciencias sociales, incluyendo antropología, criminología, educación, trabajo social, salud, ética, enfermería, transporte, entre otros. Periodical Index online es el principal índice multidisciplinario de artes, humanidades y ciencias sociales. Se incluyen más de 6.000 revistas, con alrededor de un millón de registros de nuevas revistas agregadas cada año. Las revistas completas, desde la primera edición hasta 1995, o la fecha de cese. El alcance es internacional e incluye revistas en inglés, francés, alemán, italiano, español y la mayoría de los otros idiomas occidentales. La información bibliográfica se puede buscar por separado para cada revista indexada; además, los investigadores pueden acceder a una lista de temas para cada revista y a una tabla de contenidos para cada número (www.proquest.com/products-services/periodicals_index.html).

Una de las bases más importantes es ISI Web of Knowledge, que incluye artículos de revistas, productos de conferencias internacionales, simposios, seminarios, coloquios, talleres y convenciones. Consta de tres índices de citas que cubren las ciencias sociales, las artes y las humanidades, y las ciencias. Es la fuente principal para encontrar artículos publicados en revistas arbitradas, que es donde la mayoría de la investigación se publica por primera vez. Además de proporcionar datos sobre el número de veces que los artículos publicados en una amplia gama de revistas son citados por autores de otros artículos, también entrega una fuente valiosa para identificar resúmenes y revisiones. En español, una fuente muy consultada es Teseo, base de datos de tesis doctorales españolas desde 1976. Se pueden consultar las tesis en su totalidad.

No obstante, las bases de datos no son la única fuente de literatura, y algunas veces ni siquiera son las más útiles. En muchas áreas de investigación, particularmente en las ciencias sociales, la mayor parte de la evidencia relevante puede no aparecer en revistas y se ubica en informes - en la denominada "literatura gris" -, muchos de los cuales no pueden ser indexados en bases de datos electrónicas. Por lo tanto, existe un riesgo real de que las búsquedas electrónicas por sí solas no puedan localizar una buena cantidad de información relevante. La búsqueda debe abarcar revistas, libros, capítulos de libros; de igual for- 
ma, los estudios inéditos que han circulado en ponencias, documentos de trabajo, comunicaciones privadas, tesis y trabajos de grado. Como muchos de los temas de los trabajos de grado en ciencias sociales suelen ser estudios de casos, de localidades, de regiones dentro de un país, de ciudades, o casos de liderazgos, de acciones colectivas, de elecciones locales, se sugiere no mezclar los niveles de las consultas.

Por otra parte, es muy útil y ahorra tiempo poder consultar uno o dos expertos en el tema que puedan sugerir bibliografía, incluso aportar un mapa inicial general sobre las principales fuentes (además del tutor, es pertinente consultar al menos a otra persona con experticia en el tema abordado).

III. Evaluar los estudios que serán incluidos: el mapa de la bibliografía. Una vez que se han identificado las fuentes de la bibliografía, se deben establecer criterios para la búsqueda: qué se incluye y qué se excluye. Una cuestión importante se refiere a cuántas referencias consultadas y citadas son pocas, cuántas son muchas, en fin: ¿cuántas fuentes bibliográficas son suficientes para construir un estado de la cuestión? Una respuesta general y ambigua puede ser "tantas como considere necesarias". El problema es que deja sin respuesta la pregunta y la circunscribe a una acción subjetiva y arbitraria. Otra respuesta menos general, pero que deja abierta la cuestión, es "la cantidad de fuentes bibliográficas depende del tema del trabajo de grado"; si está escribiendo sobre un campo menos estudiado, o un tema muy específico, demarcado al extremo microsocial, puede constatar que no hay muchos artículos ni libros para citar. Por el contrario, si se trata de un tema amplio, con demarcación temporal larga, macrosocial, podrá verificar que hay demasiadas publicaciones, innumerables. De manera similar, los campos más nuevos tendrán menos publicaciones a las que se pueda hacer referencia. Si es un caso especial o se trata de un tema muy poco abordado e innovador, a lo mejor no encuentra material.

Aunque algunas instituciones suelen establecer un mínimo de artículos y libros que deben incluirse en estos ejercicios, no hay un estándar universal (las bibliografías de Oxford incluyen entre 75-100 citas o fuentes; la revista Science establece un máximo de 100 referencias para los artículos de revisión; en muchas revistas internacionales se suele considerar que una revisión de un tema — que se puede asimilar a este 
ejercicio- debe contar al menos con 50 fuentes). Hay que asegurarse de haber investigado adecuadamente los documentos relevantes e incluido las citas apropiadas y, para ello, se requiere contar con criterios para determinar la cobertura de la búsqueda y de la inclusión en el documento final. Esta puede resultar una labor inaccesible, pues resulta difícil separar lo es que valioso, relevante y riguroso, de lo que no lo es. Se debe hacer un trabajo de reducción razonable de lo que se va a revisar y lo que no, pero es necesario dejar explícita la razón o las razones de la inclusión y de la exclusión. Un camino para hacer esta labor es considerar si la bibliografía detectada y seleccionada ayuda a responder las preguntas de información que se han formulado; si son pertinentes sobre la temática o no; si ha sido publicada en revistas o libros con respaldo institucional y un sistema de evaluación por pares, si está bien establecida o acreditada su calidad. No todo lo publicado amerita ser leído y reseñado, por cuanto hay numerosas publicaciones que no cuentan con procesos institucionalizados de evaluación y la calidad de sus productos puede ser discutible, o son reiteraciones, resúmenes o apuntes poco sistematizados. Una revisión exhaustiva de centenares de fuentes requiere contar con criterios de seleccióninclusión-exclusión.

Christine Susa Bruce sugiere ocho criterios para determinar la cobertura y qué textos deben ser incluidos en la revisión: la actualidad (incluir las publicaciones más recientes, casi que el último año previo al estudio, manteniendo siempre en la perspectiva de incorporación de lo que se vaya publicando); la exhaustividad (este criterio es más bien ambiguo, en todo caso debe intentar cubrir las fuentes más relevantes y hacerlo de forma sistemática, sin dejar años o publicaciones especializadas reconocidas, o libros clásicos o muy citados por fuera de la revisión y del análisis); la amplitud (ya sea en tiempo, estableciendo de forma clara un año o un periodo, así como la diversidad de fuentes a consultar); la exclusión (tener claros los criterios de lo que no se va a revisar, ya sea por capacidad logística y alcance, o por pertinencia); la relevancia (es necesario demarcar la búsqueda por la relación directa con el objeto de estudio); la circulación (por ejemplo, delimitar la búsqueda solo a revistas de circulación nacional; o solo en revistas incluidas en algún sistema y base de indexación); la disponibilidad (hay publicaciones a las que es difícil de acceder por su ubicación en alguna biblioteca fuera de alcance, o porque se trata de ejemplares ex- 
traños y escasos, o por el idioma en que fueron publicados), y la autoridad (hay artículos y libros o ponencias que, por su circulación, citas, recomendación, deben ser incluidos de forma obligada en los estados de la cuestión, otros no tanto y algunos pueden resultar irrelevantes suelen ser la mayoría-) (Bruce, 1994).

La definición de los criterios de cobertura es muy importante. Si no hay criterios claros, la búsqueda puede naufragar en el intento, pues cualquier temática cuenta con miles de referencias, o puede terminar haciendo una revisión de la literatura lejana a la temática de la cual pretende ocuparse y de la que no puede derivar nuevas cuestiones, nuevas preguntas. $\mathrm{O}$ lo contrario, si el ejercicio es arbitrario, limitado y se realiza de forma precaria, con lo que "está al alcance" puede construir un estado de la cuestión inútil, que solo le permite visualizar una porción ínfima y muy parcial de la temática. O lo puede conducir a reiterar lo que se ha hecho una o varias veces, y a lo mejor con muy buenos resultados. Es muy importante la demarcación de la temática, sin caer en la singularidad absoluta propia de algunos estudios de microhistoria, o trabajos monográficos con una espacialidad y temporalidad mínima. Como ya se expuso, es posible que, en estos ejercicios de investigación, la demarcación del objeto de estudio conduzca a que no haya trabajos previos exactamente sobre el tema, pero sí los habrá sobre temas similares en otros espacios y localidades; políticas sectoriales similares en otros momentos, países, regiones; elecciones del mismo tipo o similares; líderes y elites bajo contextos parecidos. Así, la construcción del estado de la cuestión está dirigida a conocer cómo se ha hecho antes en los mismos casos o en otros que sean comparables: cuáles teorías los han fundamentado, qué metodologías se han utilizado, qué tipos de fuentes han servido de soporte, cómo se ha conceptualizado, cuáles han sido los resultados.

IV. Organizar las fuentes y sistematizar la información. Como señala Marco Pautasso (2013), "la revisión de la literatura requiere la habilidad de hacer malabares con múltiples tareas, desde encontrar y evaluar material relevante hasta sintetizar información de varias fuentes, desde pensamiento crítico hasta parafrasear, evaluar y citar". Hay que definir claramente si se va a revisar cada pieza de la bibliografía de una forma completa, o solo se hará lectura de resúmenes o se van a combinar según las necesidades. Asimismo, es necesario contar con 
adecuadas herramientas para ir construyendo un archivo manual o electrónico de las fuentes, identificarlas, establecer su procedencia, su contenido, la pertinencia respecto de la revisión y del análisis que se está haciendo.

Para el registro se sugieren tres instrumentos sencillos: dos modelos de reseñas, uno para textos cortos y otro para obras completas, ambos son tomados de Joselyn Létourmeau y una matriz de fuentes sobre subtemas, argumentos y citas.

La tabla 1 contiene un modelo sugerido para reseñar contenidos de artículos, capítulos de libros, ponencias, reportes y otros trabajos de grado. Contiene tres partes, en la primera los diferentes ítems permiten registrar el contexto, el origen y el tipo de los textos. Los siete aspectos que contiene proveen información que permiten después organizar el texto por géneros, temas, lo que se propone, los tipos de análisis, las fuentes que utilizó el autor (esto permite, a su vez, rastrear e identificar más bibliografía) y, cuando se considere necesario, registrar también si el texto fue escrito en algún momento especial, en una coyuntura, o con motivo de un debate o de algún evento académico relevante. En la segunda parte se incluyen aspectos de contenido: tesis, su desarrollo, los conceptos y enfoques teóricos. En la tercera parte se puede incluir un balance menos apegado al texto, más procesado y reflexionado. Estos comentarios se incorporarán al balance de la bibliografía, los aportes y vacíos de la bibliografía revisada. 
Tabla 1

Modelo de reseña-ficha

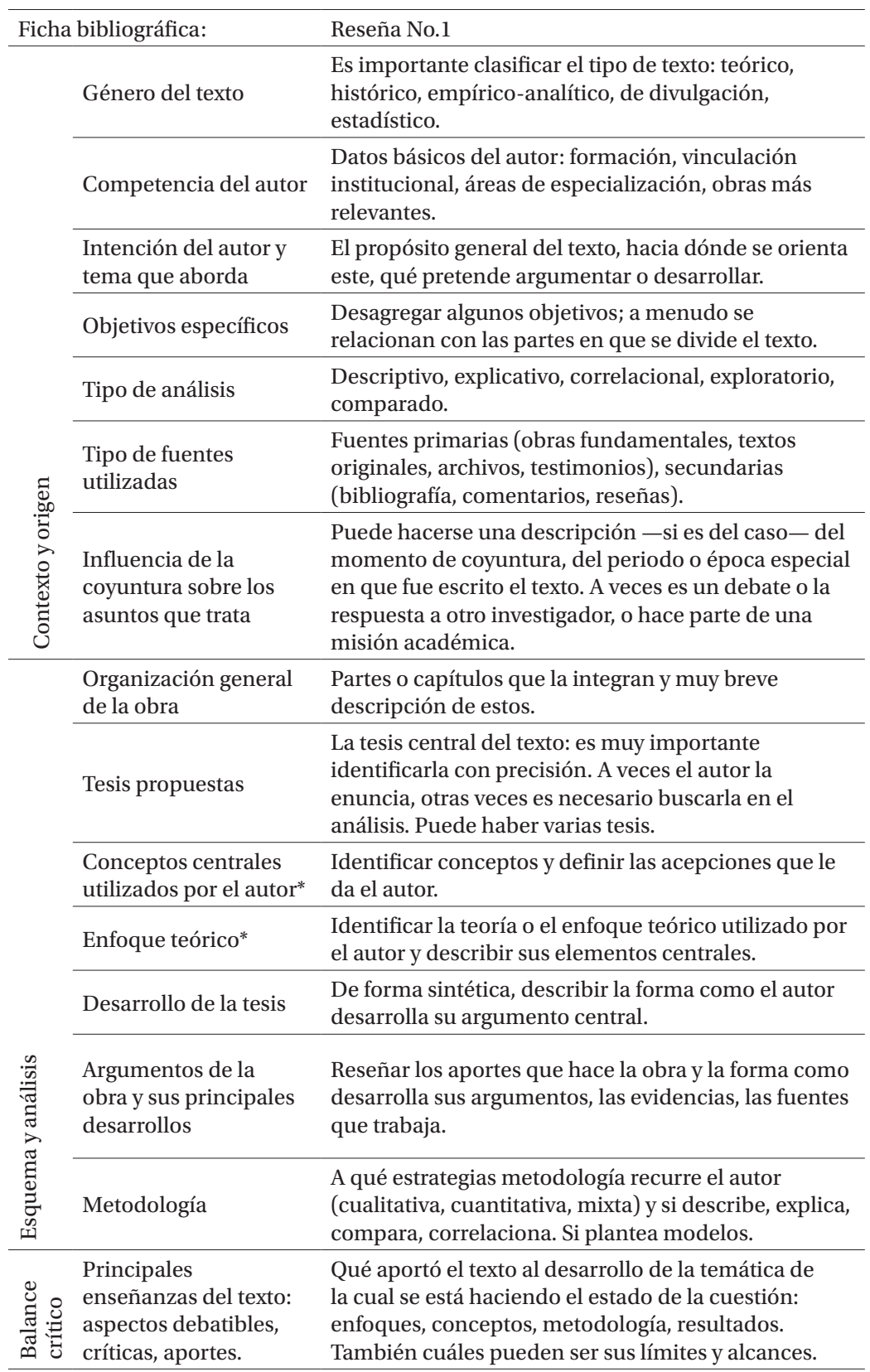

Fuente: adaptación replanteada de Létourneau (2007).

*Agregado nuestro. 
Las fichas de las reseñas proveen insumos importantes para la escritura; también permiten dar un paso adelante si se utilizan para comparar sus contenidos, los autores y las obras. La tabla 2 sugiere una forma de reunir información, plantear argumentos, comentarios y citas, de tal forma que se cuente con una base que va a servir de preescritura. La columna 1 incluye las fichas bibliográficas que sirven para identificar a los autores y su año de publicación (recordar que la cronología es importante en los estados de la cuestión), y estas se retoman de las reseñas ya hechas. La columna 2 distingue los subtemas y palabras clave que identifican el contenido específico de cada texto reseñado y considerado en la selección final de la bibliografía. En la columna 3 se incluye el o los argumentos centrales de las obras consideradas. En la columna 4 se agregan los comentarios. Estos pueden ser de diferente tipo: en algunos casos remiten a comparaciones (similitudes o diferencias con otros autores); en otros son llamados de atención por algún aporte especial, o si se trata de una obra pionera, o un texto especialmente polémico, o una novedad conceptual, teórica o metodológica. Es algo a considerar. Finalmente se incluye una columna para citas textuales que luego van a ser incorporadas en el texto escrito. No se incluyen resúmenes, tampoco otros aspectos contenidos en las fichas de las reseñas.

Tabla 2

Matriz de fuentes y de contenidos

\begin{tabular}{lllll}
\hline $\begin{array}{l}\text { Fuente (ficha } \\
\text { bibliográfica) }\end{array}$ & $\begin{array}{l}\text { Subtemas } \\
\text { (palabras } \\
\text { clave) }\end{array}$ & $\begin{array}{l}\text { Argumentos } \\
\text { centrales }\end{array}$ & Comentarios & $\begin{array}{l}\text { Citas para } \\
\text { integrar al } \\
\text { texto }\end{array}$ \\
\hline No. 1 & & & \\
\hline No.2 & & & \\
\hline No.3 & & \\
\hline No... & & \\
\hline No. 50 & & & \\
\hline
\end{tabular}

Fuente: elaboración propia.

La información contenida en las fichas y matrices de fichas constituyen los insumos básicos para el ejercicio de la escritura del estado de la cuestión. Pero no se trata de una acción mecánica de vaciamiento y ordenación de información, tampoco es un ejercicio de trascripción de fichas y de información agregada o enlistada. Como se ha analizado, de cada texto se ha registrado su contenido en las fichas y matrices; la la- 
bor complementaria es la síntesis: la capacidad de sintetizar depende de la profunda comprensión del autor sobre el tema y la bibliografía. Como lo señalan Jane Webster y Richard Watson, siempre está presente el riesgo de que los estados de la cuestión (incluso las revisiones de literatura profesionales) se conviertan en listas de citas y hallazgos, que se asemejan a una guía telefónica: muchos números pero poca trama. En contraste, una revisión coherente surge solo de una estructuración conceptual del tema. Para la mayoría de las revisiones, esto requiere de una orientación clara, basada en preguntas desagregadas de la temática, una orientación teórica y un punto de vista sobre el tema en discusión (Webster y Watson, 2002).

El análisis crítico de la literatura requiere de un examen cuidadoso de los artículos, libros, capítulos de libros, tesis, ponencias. Es importante saber ponderar los aportes de cada texto descrito y analizado. Como el propósito es tener una idea lo más completa posible del estado de conocimiento sobre el tema que se pretende indagar, no solo se debe describir el texto y resaltar sus aportes, también identificar las falencias, el nivel de desarrollo de las ideas, los faltantes, aquellas partes que han sido pobremente desarrollados en la literatura, así como inconsistencias o contradicciones entre las publicaciones sobre el tema. La crítica también identifica áreas en las que se necesitan nuevos conocimientos, a la luz de los desarrollos recientes sobre el tema, incluidas las incoherencias (Torraco, 2016).

Hay diversas alternativas para la escritura. El documento puede elaborarse con base en un orden cronológico, en cuyo caso se puede escribir sobre los materiales según cuándo estos fueron publicados. Es importante tratar de determinar momentos o etapas según el predominio de enfoques, o por giros por la publicación de una obra especial, o considerando un hecho social o político que demarca dos periodos: por ejemplo, cuando ha predominado una teoría o enfoque, las políticas públicas y la influencia del neoinstitucionalismo; o, en los países que tuvieron quiebres democráticos, se considera antes de la dictadura y posdictadura; o cuando hay un cambio institucional relevante, antes y después de la Constitución respectiva, o del cambio legislativo relevante; o antes y después de un largo periodo de hegemonía de un partido. Incluso, aunque no exista un evento significativo que permita establecer momentos o etapas, la opción del orden cronológico es válida. 
Las revisiones temáticas de la literatura se organizan también en torno a un tema o subtema, en lugar de la progresión del tiempo, de acuerdo con el predominio de ciertos temas o subtemas y su continuidad y/o discontinuidad, o el surgimiento de nuevas temáticas y el abandono de otras, o las derivaciones o desagregaciones. Incluso, es frecuente que los autores se especialicen en temas y se agrupen según este criterio. De hecho, por esta vía se tiende a romper el orden cronológico, pues deja de ser preponderante y no siempre es necesario reconstruir la bibliografía con base en el tiempo. De igual forma, se puede seguir un orden basado en la metodología utilizada en las investigaciones, y el texto se divide en apartados acordes con estas: si son más cualitativos, o cuantitativos, o testimoniales, o memorias, o estudios comparados. Depende de la temática del trabajo de grado. Puede tratarse de una temática que indaga sobre las técnicas y metodologías utilizadas en un determinado subcampo de la disciplina. La forma como son estudiados los fenómenos sociales varía en el tiempo y estas variaciones suelen estar asociadas a las metodologías que han sido utilizadas en las investigaciones, de las que puede depender el alcance o las limitaciones de sus resultados.

En síntesis, esta parte ha propuesto un camino posible, una alternativa para realizar un estado de la cuestión que incluye cuatro componentes: tener claridad sobre la temática y sobre su demarcación; determinar cómo se van a detectar e identificar las fuentes de información; evaluar los estudios que serán incluidos: el mapa de la bibliografía y organizar las fuentes y sistematizar la información. Se enfatiza: no se trata de una ruta única para hacerlo. Con el mismo espíritu del primer capítulo, es una propuesta-sugerencia que propone una forma sistemática para hacerlo.

\section{Conclusión}

Al comienzo de este artículo planteamos tres ideas que se han desarrollado en los diversos apartados en que este se divide: que los estados de la cuestión eran ejercicios académicos orientados a "usar el pasado y el presente para pensar el futuro", con base en una búsqueda, sistematización de los estudios previos y de la bibliografía procedente de diversas modalidades de publicaciones; que permiten ordenar la bibliografía en virtud de determinados criterios y reconstruir un panorama a 
partir de cosas sueltas (artículos, libros, capítulos de libros, ponencias, memorias) que se ponen en escena conectadas y comunicadas entre sí para derivar hacia nuevas búsquedas; y que de sus resultados se derivan preguntas, problemas de indagación que parten de lo que existe para proyectarse hacia lo nuevo, resultado del diagnóstico de aquello que falta, que no está bien propuesto, que denota falencias, que es incompleto, que no persuade, que inquieta.

Lo expresado en estas páginas se puede sintetizar de buena forma recurriendo al siguiente esquema de Chris Hart (1999). En el centro está el estado de la cuestión y de él se desprende componentes esenciales planteados como preguntas. Un buen ejercicio de este tipo permite tener un panorama de las teorías y conceptos centrales de la temática de la cual el estudiante se ocupa. Con la revisión de la bibliografía pertinente, seleccionada a partir de cualquiera de los criterios propuestos o combinándolos, debe quedar claro el horizonte de las teorías y conceptos clave del subcampo y de la temática respectiva. Esto requiere ya un manejo adecuado de la disciplina académica por parte del estudiante, y un proceso de aprendizaje y reaprendizaje que le permite una mayor comprensión, mayor profundidad y un cierto nivel de especialidad. En concordancia con esto, dado que se trata de un ejercicio de final de carrera y la persona está ad portas de obtener su titulación, se supone que su formación le ha permitido manejar los fundamentos epistemológicos de la disciplina, que varían de una ciencia social a otra, aunque hay aspectos compartidos.

Si el estado de la cuestión se construyó de buena forma, con una adecuada selección de obras y autores, y estos fueron debidamente "apropiados", debe haber claridad sobre los principales problemas teóricos, así como de los acuerdos y del acumulado que se ha ido construyendo en el desarrollo de la disciplina en el respectivo subcampo en que se ubica la temática. Esto supone también tener claridad sobre el origen de la problemática que se está abordando. En el desarrollo de las disciplinas hay momentos diferenciales: algunos se refieren a los comienzos y la sedimentación, otros son de mayor proyección, habrá también giros, quiebres, crisis, de igual forma que irán surgiendo nuevos problemas, aparecerán temáticas y otras irán quedando en el pasado. Es importante ubicar el contexto de origen y el devenir del desarrollo de la temática. En algunos casos se trata de temas clásicos de 
una disciplina, en otros de temas emergentes, incluso de contenidos que recién empiezan a ser abordados.

Un buen estado de la cuestión da cuenta también de los principales debates y controversias sobre la temática. Dado que todas las ciencias sociales se caracterizan por el pluralismo teórico y son "multiparadigmáticas", con frecuencia hay discusiones y confrontaciones teóricas y metodológicas sobre las diversas temáticas. El documento debe reflejar estos debates, sus argumentos, diferencias y puntos de encuentro. A la hora de realizar el trabajo de grado esto será retomado y se asumirá una postura al respecto, con base en argumentos y la evaluación de la capacidad explicativa de los enfoques y teorías.

Como lo indica el esquema, a la hora de considerar que ha adelantado en buena forma la elaboración del estado de la cuestión, cabe preguntarse ¿cómo se ha incrementado su conocimiento y entendimiento de la temática abordada? Cuando se trata de un ejercicio de investigación formativa, este constituye un pretexto de aprendizaje, una forma más de ir aprendiendo el oficio.

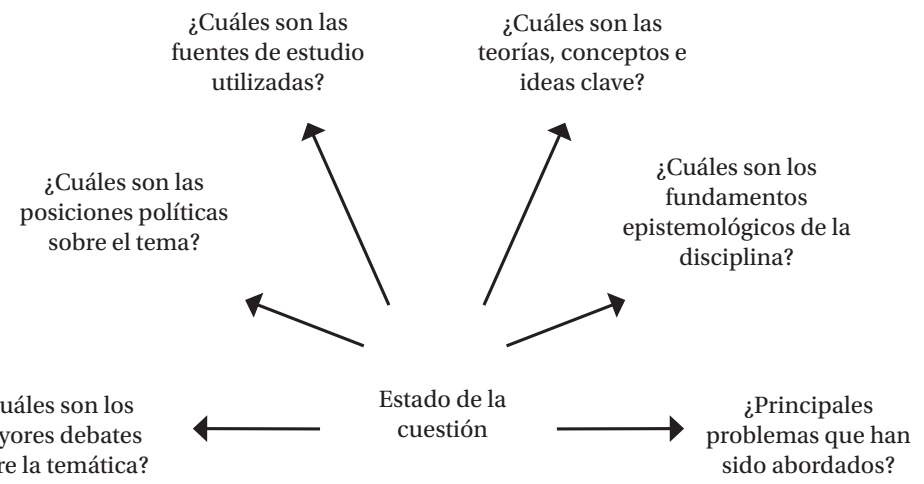

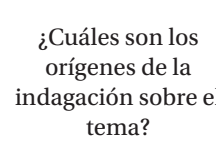

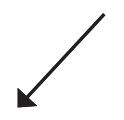
tema? ideas clave? mayores debates sobre la temática? problemas que han
sido abordados?

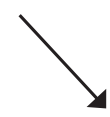

¿Cuál es el conocimiento actual del tema de indagación?

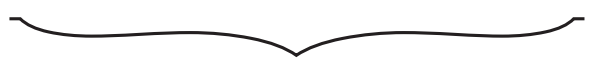

¿Cómo se ha incrementado su conocimiento y entendimiento con el estado de la cuestión?

Esquema 2. Estado de la cuestión y algunas preguntas clave 


\section{Bibliografía}

Abreo, A. M. (2004). Estado del arte de la investigación en la relación salud, niñez, desde la psicología en relación con la medicina, en Bogotá. Hallazgos, Revista de Investigaciones, 1(1), 149168. Recuperado de: http://revistas.usantotomas.edu.co/ index.php/ hallazgos/article/view/1577/1738

Arboleda, O. y Zabala, H. E. (2005). La economía solidaria en Antioquia. Estado del arte 1960-2003. Medellín: Fundación Universitaria Luis Amigó.

Baumeister, R. \& Leary, M. (1997). Writing Narrative Literature Reviews. Review of General Psychology, 1(3), 311-320.

Boote, D. \& Beile, P. (2005). Scholars Before Researchers: On the Centrality of the Dissertation Literature Review in Research Preparation. Educational Researcher, 34(6), 3-15.

Bruce, Ch. S. (1994). Research students' early experiences of the dissertation literature review. Studies in Higher Education, 19(2), 217-229.

Calvo, G. y Castro, Y. (1995). Estado del arte sobre la investigación de la familia en Colombia, Bogotá. Colombia: Ministerio de Salud, ICBF, enero de 1995. Centro de Documentación Universidad Pedagógica Nacional, CIUP-UPN.

Erren, Th., Cullen, P. \& Erren, M. (2009). How to surf today's information tsunami: On the craft of effective reading. Medical Hypotheses, 73(3): 278-279.

Galeano, M. y Vélez, O. (2002). Estado del arte sobre fuentes documentales en investigación cualitativa. Medellín: Universidad de Antioquia. Centro de Investigaciones Sociales y Humanas.

Garcés, Á., Patiño, C. y Torres, J. J. (2008). Juventud, investigación y saberes. Estado del arte de las investigaciones sobre la realidad juvenil en Medellín 2004-2006. Medellín: Universidad de Medellín.

Hart, Ch. (1999) Doing a literature review: Releasing the social science research imagination. London: Sage Publications.

Jiménez, A. (2006). El estado del arte en la investigación en ciencias sociales. En A. Jiménez y A. Torres Carrillo (Comp.), La práctica investigativa en ciencias sociales. Bogotá: Universidad Pedagógica Nacional. 
Macmillan Dictionay (s.f) State of the art. Recuperado de: https:// www.macmillandictionary.com/dictionary/british/state-ofthe-art

Maier, H. (2013). What constitutes a good literature review and why does its quality matter? Environmental Modelling and Software, (43), 3-4.

Paré, G. \& Kitsiou, S. (2017). Methods for Literature Reviews. In F. Lau and C. Kuziemsky (Eds.), Handbook of Health Evaluation: An Evidence-based Approach. University of Victory. Retrieved at https://www.ncbi.nlm.nih.gov/books/ NBK481583/

Pautasso, M. (2013). Ten Simple Rules for Writing a Literature Review, Journal PLoS Comput Biol, 9(7). Retrieved at https://www. ncbi.nlm.nih.gov/pmc/articles/PMC3715443/

Schryen, G., Wagner, G. \& Benlian, A. (2015). Theory of Knowledge for Literature Reviews: An Epistemological Model, Taxonomy and Empirical Analysis of IS Literature. Thirty Sixth International Conference on Information Systems, Fort Worth. Retrieved at https://pdfs.semanticscholar.org/c00b/617bf2324 ddef2b8175a7337ddfe838295bd.pdf

Torraco, R. (2016). Writing Integrative Literature Reviews: Using the Past and Present to Explore the Future. Human Resource Development Review, 15(4).

Urbina, A. y Morel, M. (2017). El estado del arte/estado de la técnica y la Investigación Científica y Tecnológica. Revista Portal de la Ciencia, (13), 2.

Uribe, J. (2005). La investigación documental y el estado del arte como estrategias de investigación en ciencias sociales en la investigación en ciencias sociales. Estrategias de investigación. Bogotá: Ediciones Universidad Piloto de Colombia. 